\title{
The Use of Artificial Intelligence on Colposcopy Images, in the Diagnosis and Staging of Cervical Precancers: A Study Protocol for a Randomized Controlled Trial
}

\author{
Andreea Roxana Luca ${ }^{1,2}$, Tudor Florin Ursuleanu ${ }^{1,3,4}$, Liliana Gheorghe ${ }^{1,5}$, Roxana Grigorovici ${ }^{1}$, \\ Stefan Iancu1 ${ }^{1}$, Maria Hlusneac ${ }^{1}$, Cristina Preda ${ }^{1,6}$, Alexandru Grigorovici ${ }^{1,3}$ \\ ${ }^{1}$ Faculty of General Medicine, “Grigore T. Popa” University of Medicine and Pharmacy, Iasi, Romania; ${ }^{2}$ Integrated \\ Ambulatory of Hospital "Sf. Spiridon", Department of Obstetrics and Gynecology, Iasi, Romania; "3f. Spiridon" \\ Hospital, Department of Surgery VI, Iasi, Romania; ${ }^{4}$ Regional Institute of Oncology, Iasi, Department of Surgery I, \\ Iasi, Romania; "Sf. Spiridon" Hospital, Department of Radiology, Iasi, Romania; "Sf. Spiridon” Hospital, \\ Department of Endocrinology, Iasi, Romania
}

Correspondence to: Andreea Roxana Luca, lucaandreearoxana@yahoo.com

Keywords: Artificial Intelligence, Colposcopy Images, Cervical Precancer

Received: May 17, $2021 \quad$ Accepted: June 20, $2021 \quad$ Published: June 23, 2021

Copyright $\odot 2021$ by author(s) and Scientific Research Publishing Inc.

This work is licensed under the Creative Commons Attribution International License (CC BY 4.0).

http://creativecommons.org/licenses/by/4.0/

\section{(c) (1) Open Access}

\section{ABSTRACT}

Rationale and Objectives: Accurate diagnosis and staging of cervical precancers is essential for practical medicine in determining the extent of the lesion extension and determines the most correct and effective therapeutic approach. For accurate diagnosis and staging of cervical precancers, we aim to create a diagnostic method optimized by artificial intelligence (AI) algorithms and validated by achieving accurate and favorable results by conducting a clinical trial, during which we will use the diagnostic method optimized by artificial intelligence (AI) algorithms, to avoid errors, to increase the understanding on interpretation of colposcopy images and improve therapeutic planning. Materials and Methods: The optimization of the method will consist in the development and formation of artificial intelligence models, using complicated convolutional neural networks (CNN) to identify precancers and cancers on colposcopic images. We will use topologies that have performed well in similar image recognition projects, such as Visual Geometry Group Network (VGG16), Inception deep neural network with an architectural design that consists of repeating components referred to as Inception modules (Inception), deeply separable convolutions that significantly reduce the number of parameters (MobileNet) that is a class of Convolutional Neural Network (CNN), Return of investment for machine Learning (ROI), Fully Convolutional Network (U-Net) and Overcomplete Convolutional Network Kite-Net (KiU-Net). Validation of the diagnostic method, optimized by algorithm of artificial intelligence will consist of 
achieving accurate results on diagnosis and staging of cervical precancers by conducting a randomized, controlled clinical trial, for a period of 17 months. Results: We will validate the computer assisted diagnostic (CAD) method through a clinical study and, secondly, we use various network topologies specified above, which have produced promising results in the tasks of image model recognition and by using this mixture. By using this method in medical practice, we aim to avoid errors, provide precision in diagnosing, staging and establishing the therapeutic plan in cervical precancers using AI. Conclusion: This diagnostic method, optimized by artificial intelligence algorithms and validated by the clinical trial, which we consider "second opinion", improves the quality standard in diagnosing, staging and establishing therapeutic conduct in cervical precancer.

\section{INTRODUCTION}

Colposcopy is a procedure for validating cervical lesions. Classification systems would be the Bethesda 2002 system [1] used for the classification of low-grade squamous intraepithelial lesions (LSIL) or high-grade squamous intraepithelial lesions (HSIL), formerly referred to as cervical intraepithelial neoplasia 1 (CIN1) and CIN2/CIN3 respectively. In clinical practice, the difference between LSIL and HSIL in biopsy samples is important, because in the case of high-grade lesions, the therapeutic indication is conization.

Deep learning has shown considerable potential and importance in computer diagnostics. As a gold standard for the pathological diagnosis of cervical intraepithelial lesions and invasive cervical cancer, colposcopy-guided biopsy faces challenges in improving overall accuracy and efficiency [2]. The novelty of our research consists of two elements: firstly, by the fact that we validate the computer-assisted diagnostic method through a clinical study and, secondly, we use various network topologies specified above, which have produced promising results in the tasks of image model recognition and by using this mixture we will specialize this network mix by requalifying the last layers with field-specific images-digital colposcopy. We aim to create a computer assisted diagnosis (CAD) method optimized by the algorithms of artificial intelligence and validated by accurate results on diagnosis and staging of cervical precancers and by conducting a randomized, controlled clinical trial. In our research, we will use various network topologies that have produced promising results in image model recognition tasks, such as: Visual Geometry Group Network (VGG16), Inception deep neural network with an architectural design that consists of repeating components referred to as Inception modules (Inception), deeply separable convolutions that significantly reduce the number of parameters (MobileNet) that is a class of Convolutional Neural Network (CNN), Return of investment for machine Learning (ROI), Fully Convolutional Network (U-Net) and Overcomplete Convolutional Network Kite-Net (KiU-Net) [2-4]. We will specialize these networks by requalifying the last layers with images specific to the field-digital colposcopy. We will validate the computer aided diagnosis method by the results of the clinical trial, randomized, controlled, carried out over the 17-month period. This computer assisted method not only provides automation and objectivity, but also provides a substantial benefit for women by reducing unnecessary colposcopies, in order to increase the quality standard in diagnosing, staging and establishing therapeutic conduct in cervical precancer, with defined application in gynecology [5].

\section{MATERIAL AND METHOD}

We aim to create a computer assisted diagnosis (CAD) method, optimized by the algorithms of artificial intelligence and validated by accurate results on diagnosis and staging of cervical precancers and by conducting a randomized, controlled clinical trial.

The optimization of the method will consist in development and development and pre-training of artificial intelligence algorithms for optimization the diagnosis and staging of cervical precancers through optical and imaging investigations, digital colposcopy. 
In this project we propose to tackle the problem of image pattern recognition by using a Convolutional Neural Network (CNN), as this approach has already been proved a viable instrument for this kind of tasks [2]. Being part of the larger domain of deep learning, CNN is a deep neural network, meaning that it contains multiple layers of neurons and is often used in the analysis of images. CNN is a regularized version of the classical Multilayer Perceptron (MPL), but it performs better [4]. Many times, MPL is producing a phenomenon called overfitting, meaning that it performs really well in training, but when new data is being fed to the network in the prediction phase, the results are under the desired thresholds.

By simply using a CNN for the analysis of colposcopy images with the goal of obtaining a binary (normal/abnormal) or multiclass (healthy/cervical cancer of type $1 / 2 / 3$ ) classification is not sufficient, given the particularities of the problem at hand. A naive approach could be to utilize a pre-trained CNN using a generic dataset of images such as ImageNet, Google Open Images etc., hoping that it will correctly classify the cancers. This kind of neural network, pretrained using hundreds of thousands of images, given its multilayer structure, has a very powerful of knowledge abstraction, and the naive approach must not be discarded an dis many times used as a baseline for further optimizations. We plan to use this important source of knowledge, the pretrained $\mathrm{CNN}$, although not simplistic as described above, but by applying a technique called transfer knowledge between the pretrained network and the final model.

In order to pursue this goal, in our research we will utilize various network topologies that produced promising results in image pattern recognition tasks such as VGG16, Inception or Mobilnet [4]. We will specialize the network by retraining the last few layers with domain specific images - the digital colposcopy. By using this mixed approach we will benefit of both the generic knowledge given by the pretraining of the top layers of the network against the very large image datasets and the domain specific training of the final layers, given the smaller datasets of colposcopy that we have available from our own research as well as public databases of colposcopy [6].

Moreover, in order to obtain a better score, we will apply a preprocessing method to remove specular reflection [7] and a segmentation technique to obtain a Region of Interest (ROI) to help our model to focus only on the relevant areas of the images. U-Net will be utilized, an algorithm that proved efficient $[8,9]$.

Validation of the method will consist in achieving accurate and favorable results by conducting a randomized, controlled, clinical trial, for a period of 17 months, during which we will use the diagnostic method optimized by artificial intelligence (AI) algorithms in the diagnosis and staging of cervical precancers.

Patients with cervical precancer will be included in the clinical trial. Patients will be informed and recruited through random presentation at the specialized medical centers nominated in the clinical trial and through a web platform created for this purpose. We propose the inclusion of 60 patients in the clinical trial. We'll create a control lot (G1) and a study lot (G2).

The control group (G1) will consist of patients who will be diagnosed and staging colposcopic without the use of the diagnostic method by artificial intelligence algorithms. The study group (G2) will consist of patients who will be diagnosed using the AI method and without the use of the AI method by the investigator.

Key control of diagnostic veracity staging of patients in the control group and study group will consist of the pathological anatomical outcome of the patient (biopsy/endocervical curettage, conization.

Criteria for inclusion: in the control and study groups (G1 and G2) patients with diagnosis - precancerous staging stages $0-\mathrm{IB}$, Bethesda system $[1,6]$. Exclusion criteria: in the G1 and G2 groups we exclude patients with total patient's personal pathological history (A.P.) hysterectomy, A.P. trachelectomy, pregnancy, virgo intacta, patients with Papanicolaou (Pap) smear test and negative genotyping, with comorbidities incompatible with the clinical trial.

We will use the Bethesda system [1] for the classification of cervical precancer. Colposcopic images represent by their interpretation, diagnosis and staging of precancerous cervix. We will use the binary model $0 / 1$, to note with " 0 ", lack of correspondence and " 1 " respectively correspondence, between diagnosis and staging performed by the investigator compared to the diagnosis and staging performed with the support of artificial intelligence, for cervical precancer. 
We will also use the same binary model to assess diagnostic correspondence staging of the investigator and the AI diagnostic method with anatomopathological diagnosis (biopsy/endocervical curettage, conization) and will represent the key to diagnostic control staging for the practitioner and for the AI diagnostic method. Diagnostic and staging accuracy of the AI method will result from its diagnostic correspondence with the investigator's and the anatomopathological diagnosis. Patients will be invited to sign informed standard consent to participate in the clinical trial and will be able to leave the clinical trial at any time without providing an explanation and without repercussions on treatment.

\section{RESULTS}

We will validate the computer-assisted diagnostic method through a clinical study and, secondly, we use various network topologies specified above, which have produced promising results in the tasks of image model recognition and by using this mixture we will specialize this network mix by requalifying the last layers with field-specific images, digital colposcopy. By using this method in medical practice, we aim to can avoid errors, provide precision in diagnosing, staging and establishing the therapeutic plan in cervical precancers using AI.

\section{DISCUSION}

Most computer-assisted diagnostic methods are not validated by clinical trials, and researchers have recently turned their attention to this issue. Also, the mix of deep learning architectures we use for the CAD method is a novelty. Compared to previous studies, the novelty in our research includes: creating the computer-assisted diagnostic method using network topologies mix and validate the computer-assisted diagnostic method through a clinical study. Patients will be informed, recruited through random presentation at the specialized medical centers nominated in the clinical trial and through a web platform created for this purpose. Patients will be selected on the basis of the inclusion and exclusion criteria defined in the clinical trial. The data will be processed statistically. The collection and use of patient data will be approved by the Ethics Committee on the basis of written and informed consent. By using this optimized and validated method, we aim to increase the quality standard in diagnosing, staging and establishing the therapeutic plan in precancers and cervical cancers using AI.

\section{CONCLUSION}

This diagnostic method, optimized by artificial intelligence algorithms and validated by the clinical trial, which we consider "second opinion", improves the quality standard in diagnosing, staging and establishing therapeutic conduct in cervical precancer. This CAD method develops benefits for patients reflected in improving the quality of life by maintaining sexual life, fertility, psychosomatic status and social integration induced by avoidance under or over diagnosis that can lead to delayed operational moment and implicitly to radical, disabling interventions.

\section{FUNDING}

Scientific research funded by the University of Medicine and Pharmacy "Grigore T. Popa" of Iasi, based on contract number 4714 .

\section{CONFLICTS OF INTEREST}

None declared.

\section{REFERENCES}

1. Solomon, D. Davey, D., Kurman, R., Moriarty, A., O’Connor, D., Prey, M., Raab, S., Sherman, M., Wilbur, D., Wright, T. and Young, N. (2002) The 2001 Bethesda System: Terminology for Reporting Results of Cervical 
Cytology. JAMA: The Journal of the American Medical Association, 287, 2114-2119. https://doi.org/10.1001/jama.287.16.2114

2. Ketkar, N. and Santana, E. (2017) Deep Learning with Python. Vol. 1, Springer, Berkeley, CA. https://doi.org/10.1007/978-1-4842-2766-4 1

3. Valanarasu, J.M.J., Sindagi, V.A., Hacihaliloglu, I. and Patel, V.M. (2020) Kiu-net: Overcomplete Convolutional Architectures for Biomedical Image and Volumetric Segmentation. arXiv preprint arXiv:2010.01663.

4. Driss, S.B., Soua, M., Kachouri, R. and Akil, M. (2017) A Comparison Study between MLP and Convolutional Neural Network Models for Character Recognition. SPIE Conference on Real-Time Image and Video Processing, Anaheim, CA. https://doi.org/10.1117/12.2262589

5. Wentzensen, N., Lahrmann, B., Clarke, M.A., Kinney, W., Tokugawa, D., Poitras, N., Locke, A., Bartels, L., Krauthoff, A., Walker, J., Zuna, R., Grewal, K.K., Goldhoff, P.E., Kingery, J.D., Castle, P.E., Schiffman, M., Lorey, T.S. and Grabe, N. (2021) Accuracy and Efficiency of Deep-Learning-Based Automation of Dual Stain Cytology in Cervical Cancer Screening. Journal of the National Cancer Institute, 113, 72-79.

https://doi.org/10.1093/jnci/djaa066

6. Arbyn, M., Anttila, A., Jordan, J., Ronco, G., Schenck, U., Segnan, N., Wiener, H., Herbert, A. and von Karsa, L. (2010) European Guidelines for Quality Assurance in Cervical Cancer Screening. Second Edition-Summary Document. Annals of Oncology, 21, 448-458. https://doi.org/10.1093/annonc/mdp471

7. Das, A., Kar, A. and Bhattacharyya, D. (2011) Preprocessing for Automating Early Detection of Cervical Cancer. 15th International Conference on Information Visualisation, 597-600. https://doi.org/10.1109/IV.2011.89

8. Ronneberger, O., Fischer, P. and Brox, T. (2015) U-net: Convolutional Networks for Biomedical Image Segmentation. International Conference on Medical Image Computing and Computer-Assisted Intervention, 9351, 234-241. https://doi.org/10.1007/978-3-319-24574-4 28

9. Oktay, O., Schlemper, J., Folgoc, L.L., Lee, M., Heinrich, M., Misawa, K., et al. (2018) Attention U-Net: Learning Where to Look for the Pancreas. arXiv preprint arXiv:1804.03999 